\title{
Niveles séricos de adipocitocinas y resistencia a la insulina en el primer trimestre de gestación en adolescentes y su relación con el peso del recién nacido
}

\author{
Islendy Noreña ${ }^{1,2}$, Myriam Patricia Pardo¹, Ismena Mockus ${ }^{2}$ \\ 1 Grupo de Cuidado Materno Perinatal, Facultad de Enfermería, Universidad Nacional de Colombia, Bogotá, \\ D.C., Colombia \\ 2 División de Lípidos y Diabetes, Departamento de Ciencias Fisiológicas, Facultad de Medicina, Universidad \\ Nacional de Colombia, Bogotá, D.C., Colombia
}

Introducción. Poco se ha estudiado en adolescentes gestantes la fisiología del embarazo con respecto al comportamiento de las adipocitocinas y según el modelo homeostático HOMA-IR (Homeostatic Model Assessment for Insulin Resistance) para evaluar la resistencia a la insulina y su relación con el peso del recién nacido.

Objetivo. Determinar posibles correlaciones entre las adipocitocinas leptina y adiponectina, y el HOMA-IR en adolescentes gestantes de 14 a 17 años, el índice de masa corporal en el primer trimestre de gestación y el peso del recién nacido.

Materiales y métodos. En las semanas 11 a 14 de gestación, se midieron las variables bioquímicas de la leptina y la adiponectina, así como de la glucemia y la insulina, y se calculó el puntaje del HOMA-IR. Se obtuvieron los datos sobre las variables antropométricas de las madres y los recién nacidos. En el análisis estadístico se calcularon la correlación de Pearson y el valor de $p$.

Resultados. Se evidenció una correlación positiva entre los niveles séricos de la leptina y el HOMA-IR en el primer trimestre de gestación $(r=0,5 ; p \leq 0,000)$ y una negativa entre la adiponectina y el HOMAIR $(r=-0,4 ; p=0,017)$, además de correlaciones positivas del índice de masa corporal con la leptina, la insulina y el HOMA-IR ( $r=0,83$ y $p<0,000 ; r=0,56$ y $p=<0.000$, y $r=0,54$ y $p \leq 0,000$, respectivamente). En madres adolescentes sin obesidad ni antecedentes de dislipidemia, se registró una correlación positiva entre la evaluación HOMA-IR y el peso neonatal ( $r=0,43 ; p=0,012)$.

Conclusiones. La leptina y el HOMA-IR presentaron una correlación positiva, y la adiponectina y el HOMA-IR, una negativa. La leptina y el HOMA-IR se correlacionaron de manera positiva con el IMC. EI HOMA-IR se correlacionó con el peso de los recién nacidos de adolescentes sin obesidad ni dislipidemia.

Palabras clave: resistencia a la insulina; leptina; adiponectina; embarazo en adolescencia; recién nacido; peso al nacer.

doi: https://doi.org/10.7705/biomedica.v38i4.4035

Serum adipokine levels and insulin resistance in the first trimester of pregnancy in adolescents and their relationship with neonatal weight

Introduction: The approach to the physiology of pregnancy based on adipokine behavior and the homeostasis-insulin resistance (HOMA-IR) model, along with their relationship to neonatal weight, has been poorly studied in adolescent pregnant women.

Objective: To determine possible correlations between adipokines -leptin and adiponectin- and HOMA-IR in pregnant women aged 14 to 17 years, and first-trimester body mass index (BMI) and neonatal weight.

Materials and methods: In the weeks 11 to 14 of gestation, the biochemical variables leptin, adiponectin, glycemia and insulin were measured and HOMA-IR was calculated. Maternal and neonatal anthropometric variables were obtained. Statistical analysis was performed with Pearson correlation and the $p$ value.

Results: We noticed a positive correlation of serum leptin levels with HOMA-IR in the first trimester of gestation $(r=0.5, p \leq 0.000)$ and a negative correlation between adiponectin and HOMA-IR $(r=-0.4$; $p=0.017)$, along with positive correlations between BMI and leptin, insulin and HOMA-IR $(r=0.83$ and

\section{Contribución de los autores:}

Islendy Noreña e Ismena Mockus: concepción y diseño del estudio, análisis e interpretación de resultados Islendy Noreña y Myriam Patricia Pardo: recolección y consolidación de datos

Todas las autoras participaron en la escritura del manuscrito. 
$p<0.000, r=0.56$ and $p \leq 0.000 ; r=0.54$ and $p \leq 0.000$, respectively). In adolescent non-obese mothers with no history of dyslipidemia, there was a positive correlation between HOMA-IR and neonatal weight $(\mathrm{r}=0.43, \mathrm{p}=0.012)$.

Conclusions: Leptin and HOMA-IR showed a positive correlation, while adiponectin and HOMA-IR showed a negative correlation. Leptin and HOMA-IR were positively correlated with BMI. HOMA-IR correlated with the weight of neonates of non-obese adolescents without dyslipidemia.

Key words: Insulin resistance; leptin; adiponectin; pregnancy in adolescence; infant, newborn; birth weight.

doi: https://doi.org/10.7705/biomedica.v38i4.4035

El embarazo en mujeres adolescentes es de interés para la salud pública y la ginecoobstetricia. En su estudio prima el componente nutricional, ya que sus alteraciones se asocian con complicaciones durante el periodo prenatal, el parto y el posparto, lo que aumenta los riesgos de un mal resultado obstétrico.

Por una parte, las mujeres gestantes con alteraciones nutricionales, como la obesidad, presentan un mayor riesgo de aborto y una disminución de las tasas de implantación embrionaria, así como predisposición a padecer síndromes hipertensivos, diabetes de la gestación, tromboembolia, infecciones puerperales y complicaciones de las heridas quirúrgicas. En el recién nacido, hay un mayor riesgo de macrosomía o bajo peso, hipoglucemia y de anomalías congénitas, que conllevan hospitalizaciones y estancias prolongadas en la unidad neonatal de cuidados intensivos (1).

Por otra parte, la deficiencia de hierro y la anemia en la gestación se asocian con resultados desfavorables para el feto y el desarrollo del embarazo, tales como retraso en el crecimiento intrauterino, parto prematuro, bajo peso y disminución de la respuesta inmunológica del recién nacido (2). En el 2015 en Colombia hubo un porcentaje mayor de estos casos en las madres menores de 20 años (7,8 \% Vs. $7,6 \%$ en madres entre los 20 y los 34 años; y 7,5 \% en madres de 35 a 49 años) (3).

Es importante centrar la atención en las mujeres adolescentes gestantes, dado que en nuestro país la incidencia de estos casos sigue siendo elevada. La menarquía temprana, el pronto inicio de relaciones sexuales, los bajos niveles educativo y socioeconómico, el desconocimiento y la no utilización de métodos anticonceptivos constituyen factores de riesgo para el embarazo adolescente (3).

\footnotetext{
Correspondencia:

Islendy Noreña, calle 73 A № 83-16, Bogotá, D.C., Colombia

Teléfonos: (57) 3917918 y (314) 2369203

inorenaa@unal.edu.co

Recibido: 16/08/17; aceptado: 02/04/18
}

Según el Fondo de Población de las Naciones Unidas (UNFPA), la región de Latinoamérica y el Caribe presenta la segunda tasa más alta de embarazos del mundo, y se estima que el $15 \%$ de los nacimientos corresponde a mujeres menores de 20 años (4). Según la Encuesta Nacional de Demografía y Salud (ENDS, 2015), la tasa específica de fecundidad en mujeres adolescentes colombianas entre los 15 y los 19 años es de 17,4\%. Además, las mujeres adolescentes gestantes son un grupo de alto riesgo debido a sus necesidades nutricionales, las cuales difieren de las de la mujer adulta; en las adolescentes, se conjugan las necesidades nutricionales para su propio crecimiento con aquellas para el desarrollo fetal (5).

Es bien conocido que el embarazo se asocia con cambios en la ingestión de alimentos, la grasa corporal, el gasto energético y la resistencia a la insulina; además, en varios estudios se ha establecido que las adipocitocinas (leptina y adiponectina) juegan un papel importante en la regulación del metabolismo materno y, en algunos, se sugiere que la leptina guarda relación con la grasa corporal materna y el índice HOMAIR (Homeostatic Model Assessment for Insulin Resistance), modelo homeostático para evaluar la resistencia a la insulina (6).

Durante el embarazo se genera un estado de resistencia a la leptina asociado con disminución de su señalización en el hipotálamo, cuya posible función es mejorar la movilización de las reservas maternas de grasa para aumentar la disponibilidad de sustratos lipídicos e incrementar su transferencia transplacentaria (7).

En diversos artículos sobre las mujeres adolescentes gestantes, se ha descrito que en la adolescencia aumenta la concentración de leptina asociada con el aumento del tejido adiposo, y el inicio de la menarquia y la función reproductiva. Durante la gestación en las adolescentes, el incremento de la leptina se ha relacionado con retardo del crecimiento intrauterino y un mayor riesgo de bajo peso en el recién nacido (8). 
La secreción de adiponectina, por su parte, disminuye gradualmente a medida que avanza la gestación. Estos niveles bajos pueden jugar un papel en el aumento de la resistencia a la insulina, aspecto que favorece la provisión de glucosa para el feto. La adiponectina se correlaciona negativamente con la masa grasa $y$, a medida que aumenta el tejido adiposo, disminuyen los niveles séricos de esta adipocitocina y aumenta la reserva energética para el feto y la placenta (7).

En estudios previos se han explorado las posibles correlaciones de estos biomarcadores séricos con algunas variables antropométricas fetales. En Chile se llevó a cabo un estudio prospectivo de casos y controles en adultas gestantes que se encontraban entre las semanas 11 y 14 de gestación para determinar la relación entre los niveles de adiponectina en el primer trimestre de gestación y el retardo del crecimiento fetal; se observó que, cuando los niveles de adiponectina eran mayores en el primer trimestre, el recién nacido podía presentar bajo peso (9).

En cuanto a la resistencia a la insulina, Crume, et al., estudiaron las relaciones entre los niveles circulantes de glucosa y lípidos en la madre, y la resistencia a la insulina en la primera y la segunda mitad de la gestación y la composición corporal neonatal. Encontraron una correlación positiva entre el HOMA-IR de la primera mitad de la gestación con la masa grasa neonatal, independientemente del índice de masa corporal (IMC) al comienzo de la gestación. En la segunda mitad de la gestación, se presentó una correlación positiva entre los niveles de glucosa materna y el porcentaje de masa grasa fetal (10).

El desequilibrio entre el consumo y el gasto de energía puede influir en la duración del periodo de gestación y en el peso del recién nacido. Los recién nacidos pequeños o grandes para la edad de gestación se enfrentan a un mayor riesgo de mortalidad y a una variedad de problemas de salud, incluidas las enfermedades metabólicas y cardiovasculares en la edad adulta (11).

La fisiología del embarazo con respecto al comportamiento de las adipocitocinas leptina y adiponectina, el de la insulina y la evaluación HOMA-IR, se ha estudiado principalmente en mujeres adultas gestantes, pero poco en adolescentes gestantes. En este sentido, en este estudio se propuso determinar posibles correlaciones entre las adipocitocinas leptina y adiponectina, el IMC y el puntaje de el HOMA-IR del primer trimestre de gestación en adolescentes entre 14 y 17 años, así como analizar las posibles correlaciones entre los biomarcadores séricos mencionados y el HOMA-IR con el peso del recién nacido.

\section{Materiales y métodos}

Se hizo un estudio exploratorio, longitudinal, observacional y prospectivo que contó con el aval del Comité de Ética de la Facultad de Medicina de la Universidad Nacional de Colombia.

Se incluyeron mujeres adolescentes de 14 a 17 años con 11 a 14 semanas de su primera gestación confirmada por ecografía, después de la firma del consentimiento y el asentimiento informados. Se descartaron los casos de adolescentes con discapacidades cognitivas, enfermedad mental, síndrome convulsivo, hipotiroidismo o hipertensión arterial previos al embarazo, así como los de embarazo gemelar. La muestra estuvo constituida por 40 adolescentes gestantes captadas en cuatro hospitales públicos de Bogotá.

Las participantes fueron citadas en ayunas en compañía de un acudiente a los respectivos centros de salud, donde asistían a los controles prenatales. Después de explicarles los objetivos y el alcance de la investigación, así como los procedimientos, y tras la firma de los consentimientos y asentimientos informados, se procedió a medir y a tomar la talla de cada participante para calcular el IMC. Se utilizaron una balanza mecánica (Healthometer ${ }^{\circledR}$ ) y una cinta métrica. Cada participante debía estar descalza, con ropa ligera y en posición erguida en el momento de ser pesada, y para la talla, debía estar descalza, en posición erguida, con la mirada al frente, talones juntos y rodillas rectas contra una superficie lisa.

Como valores de referencia del peso materno, se tuvieron en cuenta los rangos establecidos por la Organización Mundial de la Salud (OMS) y adoptados por el Ministerio de Salud y Protección Social de Colombia en la Resolución 00002465 de 2016: IMC bajo: menor de $20 \mathrm{~kg} / \mathrm{m}^{2}$; IMC adecuado: 20 a $24,9 \mathrm{~kg} / \mathrm{m}^{2}$; sobrepeso: 25 a 29,9 $\mathrm{kg} / \mathrm{m}^{2}$; obesidad: igual o mayor a $30 \mathrm{~kg} / \mathrm{m}^{2}(12)$.

Se consideró el peso del niño al nacer registrado en la historia clínica confirmado por el reporte verbal de la madre adolescente. Se tuvieron en cuenta los siguientes rangos: bajo peso al nacer: inferior a $2.500 \mathrm{~g}$; peso normal: 2,500 a $4.000 \mathrm{~g}$; peso alto: mayor de $4.000 \mathrm{~g}(13,14)$. 
Para las mediciones bioquímicas, se obtuvieron $10 \mathrm{ml}$ de sangre venosa de la vena antecubital y, después de la centrifugación, los sueros se dividieron en tres alícuotas. Dos se almacenaron a $-80^{\circ} \mathrm{C}$ para determinar los niveles séricos de leptina, adiponectina e insulina, utilizando una prueba ELISA $\left(\right.$ R\&D Systems $\left.{ }^{\circledR}\right)$, y la otra se almacenó entre 2 y $8{ }^{\circ} \mathrm{C}$ para determinar la glucemia.

El índice del modelo homeostático para evaluar la resistencia a la insulina HOMA-IR, se calculó mediante la siguiente fórmula: (glucemia basal $(\mathrm{mmol} / \mathrm{L}) \times$ insulina basal $(\mu \mathrm{Ul} / \mathrm{ml})) / \mathrm{k}(\mathrm{k}=22,5)$. Se recolectó la información de la atención del parto sobre la edad en semanas de gestación al nacer, el tipo de parto, el peso y la talla del recién nacido.

Los datos se analizaron mediante el programa estadístico SPSS ${ }^{\mathrm{TM}}$, versión 12. Las correlaciones entre las diferentes variables bioquímicas y antropométricas, se obtuvieron mediante el coeficiente de correlación de Pearson y, para la evaluación de las pruebas de hipótesis, se empleó la prueba de ji al cuadrado.

\section{Resultados}

Las adolescentes gestantes se clasificaron según el IMC en el momento de ingreso en el estudio. Hubo 19 (47\%) con un IMC menor de $20 \mathrm{~kg} / \mathrm{m}^{2}$, $16(40 \%)$ con un IMC normal, 4 (10\%) con un IMC entre 25 y $29,9 \mathrm{~kg} / \mathrm{m}^{2}$, y 1 (3\%) con uno mayor de
$30 \mathrm{~kg} / \mathrm{m}^{2}$. En el cuadro 1 se presenta la caracterización general de las adolescentes gestantes y se relacionan las medidas de tendencia central del IMC, los biomarcadores séricos, el índice HOMA$\mathrm{IR}$, y el peso y la talla de los recién nacidos.

Con el fin de evaluar la relación entre los biomarcadores séricos (leptina, adiponectina, insulina, glucemia) y el IMC inicial de las participantes, se utilizó el coeficiente de correlación de Pearson y sus correspondientes inferencias, así como la prueba de ji al cuadrado para determinar las relaciones significativas (cuadro 2). Se encontró una correlación positiva estadísticamente significativa $(p<0,0000)$ entre los niveles circulantes de leptina $y$ el IMC inicial $(r=0,839)$. Asimismo, se observó una correlación positiva entre el IMC inicial y los niveles de insulina $(r=0,56 ; p \leq 0,0001)$, y entre el índice HOMA-IR y el IMC inicial $(r=0,54 ; p=0,0003)$.

En el cuadro 3 se presentan las correlaciones entre los biomarcadores séricos del primer trimestre de gestación. Las correlaciones positivas significativas relacionadas con los niveles séricos de leptina, fueron: leptina e IMC inicial $(r=0,8$; $p<0,000)$, leptina e insulina $(r=0,6 ; p=0,0001)$, y leptina y el índice HOMA-IR ( $r=0,5 ; p=0,0002)$. En cuanto a los niveles séricos de la insulina, se encontraron las siguientes correlaciones positivas significativas: insulina e IMC inicial $(r=0,6$; $p=0,0002)$, e insulina y glucemia basal $(r=0,5$;

Cuadro 1. Caracterización de las adolescentes gestantes en estudio

\begin{tabular}{|c|c|c|c|c|c|c|c|c|}
\hline & $\mathbf{n}$ & Media & $\begin{array}{c}\text { Desviación } \\
\text { estándar }\end{array}$ & Mínimo & Máximo & $\begin{array}{c}\text { Percentil } \\
25\end{array}$ & $\begin{array}{c}\text { Percentil } \\
50\end{array}$ & $\begin{array}{c}\text { Percenti } \\
75\end{array}$ \\
\hline IMC inicial $\left(\mathrm{kg} / \mathrm{m}^{2}\right)$ & 40 & 21,1 & 3,4 & 14,7 & 33,6 & 19,2 & 20,4 & 22,7 \\
\hline Leptina (ng/ml) & 40 & 24,4 & 22,5 & 3,3 & 115,1 & 10,0 & 17,8 & 29,3 \\
\hline Adiponectina $(\mu \mathrm{g} / \mathrm{ml})$ & 40 & 13,1 & 6,0 & 3,0 & 29,8 & 9,0 & 13,0 & 16,6 \\
\hline Insulina $(\mu \mathrm{Ul} / \mathrm{ml})$ & 40 & 8,4 & 5,4 & 2,2 & 30,1 & 5,8 & 7,0 & 9,8 \\
\hline Glucemia basal (mg/dl) & 40 & 82,3 & 7,2 & 68,0 & 97,0 & 77,7 & 81,4 & 87,4 \\
\hline HOMA-IR $(\mathrm{mmol} / \mathrm{L} \times \mu \mathrm{Ul} / \mathrm{ml} / \mathrm{k})$ & 40 & 1,7 & 1,3 & 0,4 & 7,1 & 1,1 & 1,5 & 2,0 \\
\hline Peso neonatal $(\mathrm{g})$ & 39 & $2.937,9$ & $5.33,9$ & $1.410,0$ & $3.770,0$ & $2.665,0$ & $3.010,0$ & $3.270,0$ \\
\hline Talla neonatal $(\mathrm{cm})$ & 39 & 49,6 & 3,4 & 38,0 & 56,0 & 49,0 & 50,0 & 51,0 \\
\hline
\end{tabular}

IMC: índice de masa corporal; HOMA-IR: modelo homeostático para evaluar la resistencia a la insulina (Homeostatic Model Assessment for Insulin Resistance, HOMA-IR)

Cuadro 2. Correlaciones entre el índice de masa corporal inicial, los biomarcadores séricos y el índice HOMA-IR

\begin{tabular}{|c|c|c|c|c|c|c|c|c|c|c|}
\hline & $\begin{array}{c}\mathrm{CT} \\
\mathrm{mg} / \mathrm{dl}\end{array}$ & $\begin{array}{l}\mathrm{C}-\mathrm{LDL} \\
\mathrm{mg} / \mathrm{dl}\end{array}$ & $\begin{array}{l}\mathrm{c}-\mathrm{HDL} \\
\mathrm{mg} / \mathrm{dl}\end{array}$ & $\begin{array}{l}\text { TGC } \\
\mathrm{mg} / \mathrm{dl}\end{array}$ & $\begin{array}{c}\text { Leptina } \\
\mathrm{ng} / \mathrm{ml}\end{array}$ & $\begin{array}{c}\text { Adiponectina } \\
\mu \mathrm{g} / \mathrm{ml}\end{array}$ & $\begin{array}{c}\text { Insulina } \\
\mu \mathrm{ui} / \mathrm{ml}\end{array}$ & $\underset{\mathrm{mg} / \mathrm{dl}}{\text { Glucemia }}$ & $\begin{array}{c}\text { HOMA - IR } \\
(\mathrm{mmol} / \mathrm{l} \times \mu \mathrm{UI} / \mathrm{ml} / \mathrm{k})\end{array}$ & $\begin{array}{l}\text { IMC } \\
\text { inicial }\end{array}$ \\
\hline \multicolumn{11}{|l|}{ IMC inicial } \\
\hline Correlación de Pearson & 0,166 & 0,037 & 0,123 & 0,013 & 0,839 & $-0,124$ & 0,56 & 0,196 & 0,54 & 1 \\
\hline Sig. (bilateral) & 0,306 & 0,820 & 0,449 & 0,937 & 0,0000000001 & 0,448 & 0,00017 & 0,225 & 0,00033 & \\
\hline $\mathrm{N}$ & 40 & 40 & 40 & 40 & 40 & 40 & 40 & 40 & 40 & 40 \\
\hline
\end{tabular}

IMC: índice de masa corporal; HOMA-IR: modelo homeostático para evaluar la resistencia a la insulina (Homeostatic Model Assessment for Insulin Resistance, HOMA-IR); k=22,5; CT: colesterol total; TGC: trigliceridos 
Cuadro 3. Correlaciones entre los biomarcadores séricos en el primer trimestre de gestación

\begin{tabular}{|c|c|c|c|c|c|c|c|c|c|c|c|c|}
\hline & & $\begin{array}{c}\mathrm{CT} \\
(\mathrm{mg} / \mathrm{dl})\end{array}$ & $\begin{array}{l}\text { Colesterol } \\
\text { HDL (mg/dl) }\end{array}$ & $\begin{array}{l}\text { Colesterol } \\
\text { LDL (mg/dl) }\end{array}$ & $\begin{array}{l}\mathrm{TGC} \\
(\mathrm{mg} / \mathrm{dl})\end{array}$ & $\begin{array}{l}\text { Leptina } \\
\text { (ng/ml) }\end{array}$ & $\begin{array}{c}\text { Adiponectina } \\
(\mu \mathrm{g} / \mathrm{ml})\end{array}$ & $\begin{array}{l}\text { Insulina } \\
(\mu \mathrm{Ul} / \mathrm{ml})\end{array}$ & $\begin{array}{c}\text { Glucemia } \\
\text { basal } \\
\text { (mg/dl) }\end{array}$ & $\begin{array}{l}\text { HOMA-IR } \\
(\mathrm{mmol} / \mathrm{L} \mathrm{x} \\
\mu \mathrm{Ul} / \mathrm{ml} / \mathrm{k})\end{array}$ & $\begin{array}{c}\text { IMC } \\
\text { inicial } \\
\left(\mathbf{k g} / \mathbf{m}^{2}\right)\end{array}$ & $\begin{array}{c}\text { IMC final } \\
\left(\mathrm{kg} / \mathrm{m}^{2}\right)\end{array}$ \\
\hline \multirow{3}{*}{ Leptina } & Cor & 0,3 & 0,1 & 0,2 & $-0,1$ & 1.0 & $-0,1$ & 0,6 & 0,1 & 0,5 & 0,8 & 0,6 \\
\hline & Sig. & 0,1 & 0,5 & 0,2 & 0,7 & & 0,6 & 0,000 & 0,4 & 0,000 & $0,0^{*}$ & 0,000 \\
\hline & $\mathrm{N}$ & 40,0 & 40,0 & 40,0 & 40,0 & 40,0 & 40,0 & 40,0 & 40,0 & 40,0 & 40,0 & 40,0 \\
\hline \multirow[t]{3}{*}{ Adiponectina } & Cor & 0,4 & 0,4 & 0,2 & 0,0 & $-0,1$ & 1,0 & $-0,4$ & $-0,3$ & $-0,4$ & $-0,1$ & 0,0 \\
\hline & Sig. & 0,008 & 0,01 & 0,3 & 1,0 & 0,6 & & 0,025 & 0,048 & 0,017 & 0,4 & 0,9 \\
\hline & $\mathrm{N}$ & 40,0 & 40,0 & 40,0 & 40,0 & 40,0 & 40,0 & 40,0 & 40,0 & 40,0 & 40,0 & 40,0 \\
\hline \multirow[t]{3}{*}{ Insulina } & Cor & 0,0 & $-0,2$ & 0,0 & 0,1 & 0,6 & $-0,4$ & 1,0 & 0,5 & 1,0 & 0,6 & 0,5 \\
\hline & Sig. & 0,9 & 0,2 & 0,8 & 0,6 & 0,0001 & 0,0254 & & 0,003 & 0,0 & 0,000 & 0,003 \\
\hline & $\mathrm{N}$ & 40,0 & 40,0 & 40,0 & 40,0 & 40,0 & 40,0 & 40,0 & 40,0 & 40,0 & 40,0 & 40,0 \\
\hline \multirow{3}{*}{$\begin{array}{l}\text { Glucemia } \\
\text { basal }\end{array}$} & Cor & 0,0 & $-0,3$ & 0,1 & 0,0 & 0,1 & $-0,3$ & 0,5 & 1,0 & 0,5 & 0,2 & 0,2 \\
\hline & Sig. & 0,9 & 0,1 & 0,7 & 0,9 & 0,4 & 0,0484 & 0,0030 & & 0,000 & 0,2 & 0,2 \\
\hline & $\mathrm{N}$ & 40,0 & 40,0 & 40,0 & 40,0 & 40,0 & 40,0 & 40,0 & 40,0 & 40,0 & 40,0 & 40,0 \\
\hline \multirow{3}{*}{ HOMA-IR } & Cor & 0,0 & $-0,2$ & 0,0 & 0,1 & 0,5 & $-0,4$ & 1,0 & 0,5 & 1,0 & 0,5 & 0,5 \\
\hline & Sig. & 1,0 & 0,2 & 0,9 & 0,6 & 0,0002 & 0,0175 & 0,00 & 0,0003 & & 0,000 & 0,003 \\
\hline & $\mathrm{N}$ & 40,0 & 40,0 & 40,0 & 40,0 & 40,0 & 40,0 & 40,0 & 40,0 & 40,0 & 40,0 & 40,0 \\
\hline \multirow{3}{*}{ IMC inicial } & Cor & 0,2 & 0,1 & 0,0 & 0,0 & 0,8 & $-0,1$ & 0,6 & 0,2 & 0,5 & 1,0 & 0,9 \\
\hline & Sig. & 0,3 & 0,4 & 0,8 & 0,9 & $0,0^{*}$ & 0,4 & 0,0002 & 0,2 & 0,0003 & & $0,0^{*}$ \\
\hline & $\mathrm{N}$ & 40,0 & 40,0 & 40,0 & 40,0 & 40,0 & 40,0 & 40,0 & 40,0 & 40,0 & 40,0 & 40,0 \\
\hline
\end{tabular}

Las correlaciones marcadas con asterisco $\left(^{*}\right)$ indican un valor de $p<0,00000$. CT: colesterol total; TGC: triglicéridos

$p=0,0030)$. También se encontró una correlación positiva significativa entre el índice HOMA-IR y el IMC inicial $(r=0,5 ; p=0,0003)$.

Las siguientes fueron las correlaciones negativas más importantes: adiponectina e índice HOMA-IR $(r=-0,4 ; p=0,0175)$, adiponectina e insulina $(r=-0,4$; $\mathrm{p}=0,0254)$, y adiponectina y glucemia basal ( $r=-$ $0,3 ; p=0,0484)$. No se encontraron correlaciones de los niveles séricos de colesterol total (HDL y LDL) y de los triglicéridos con el peso del recién nacido.

En cuanto a las correlaciones entre los biomarcadores séricos del primer trimestre de gestación y el peso del recién nacido, se descartó a una paciente, ya que en la semana 18 de gestación presentó un aborto espontáneo. Además, se descartaron tres díadas debido a las siguientes alteraciones maternas: antecedente de hipertrigliceridemia y estado prehipertensivo en el momento del parto; obesidad $\left(\mathrm{IMC}=33 \mathrm{~kg} / \mathrm{m}^{2}\right)$ y parto prematuro, y sobrepeso $\left(\mathrm{IMC}=27,5 \mathrm{~kg} / \mathrm{m}^{2}\right)$, con retardo del crecimiento intrauterino y prematuridad. Las tres díadas madrehijo se retiraron del estudio porque las alteraciones asociadas con la hipertrigliceridemia, el sobrepeso y la obesidad producen cambios significativos en el comportamiento de las adipocitocinas y el índice HOMA-IR, y constituyen un riesgo de malos resultados obstétricos $(15,16)$.

En el cuadro 4 se presentan las semanas de gestación en el momento del nacimiento, el peso de los recién nacidos y el índice HOMA-IR del primer trimestre de gestación de las díadas definitivas del estudio y, en el cuadro 5 se presenta la correlación positiva entre el índice HOMA-IR y el peso de los recién nacidos $(r=0,43 ; p=0,012)$.

\section{Discusión}

Los resultados evidenciaron correlaciones positivas entre los niveles circulantes de los biomarcadores séricos y el IMC inicial. Entre las principales correlaciones está el IMC inicial y la leptina $(r=0,839$; $\mathrm{p}<0,000)$; esta misma correlación se halló en las mediciones de la leptina en el primer trimestre de gestación de mujeres adultas en el estudio de Kac, et al. (17), en Brasil, y en el estudio de Walsh (18) en Irlanda. En estudios previos se ha determinado que los niveles séricos de la leptina, adipocitocina producida principalmente por los adipocitos y la placenta, aumentan simultáneamente con el peso durante la gestación (19-21).

El IMC y los valores séricos de insulina en el primer trimestre de gestación, presentaron una correlación positiva significativa $(r=0,56 ; p=0,00017)$, lo que coincide con los hallazgos obtenidos en los estudios de estos autores $(17,18)$. También, el IMC inicial y el índice HOMA-IR presentaron una correlación positiva significativa $(r=0,54 ; p=0,00033)$, resultados afines a los reportados en el estudio de Kac, et al. (17). Esta correlación puede deberse a que, ante una mayor presencia de masa grasa, aumenta el índice HOMA-IR, indicador de resistencia a la insulina, como en el caso de individuos con un IMC alto. 
El aumento del tamaño del adipocito desencadena la secreción de la proteína quimioatrayente de monocitos (MCP-1), la cual conlleva la infiltración de macrófagos y secretores de citocinas inflamatorias, como el factor de necrosis tumoral alfa (TNF-a) y la interleucina 6, que alteran la vía de señalización de la insulina mediante varios mecanismos, entre ellos, la inhibición de la fosforilación en tirosinas de los sustratos del receptor de la insulina (IRS) (22-24). En últimas, disminuye

Cuadro 4. Edad de gestación al nacer, valores del peso del recién nacido y del índice HOMA-IR materno en el inicio del embarazo

\begin{tabular}{|c|c|c|}
\hline $\begin{array}{l}\text { Edad de gestación al } \\
\text { nacer (semanas) }\end{array}$ & $\begin{array}{l}\text { Peso del recién } \\
\text { nacido }(\mathrm{g})\end{array}$ & $\begin{array}{l}\text { HOMA-IR materno } \\
(\mathrm{mmol} / \mathrm{L} \times \mu \mathrm{ui} / \mathrm{ml} / \mathrm{k})\end{array}$ \\
\hline 35,6 & 1.470 & 0,71 \\
\hline 33,4 & 2.060 & 2,01 \\
\hline 36,3 & 2.310 & 1,21 \\
\hline 37,6 & 2.350 & 1,44 \\
\hline 39 & 2.420 & 0,87 \\
\hline 38 & 2.550 & 0,63 \\
\hline 40 & 2.660 & 1,2 \\
\hline 37,1 & 2.665 & 1,57 \\
\hline 37 & 2.675 & 1,23 \\
\hline 38 & 2.780 & 0,39 \\
\hline 37,6 & 2.800 & 1,43 \\
\hline 38,5 & 2.800 & 1,47 \\
\hline 40,2 & 2.810 & 1,12 \\
\hline 40 & 2.860 & 1,62 \\
\hline 37,3 & 2.880 & 1,17 \\
\hline 39,1 & 2.930 & 1,28 \\
\hline 37 & 2.980 & 1,03 \\
\hline 40 & 3.010 & 1,94 \\
\hline 40 & 3.070 & 1,46 \\
\hline 39,6 & 3.100 & 1,59 \\
\hline 40 & 3.100 & 2,27 \\
\hline 39 & 3.120 & 1,26 \\
\hline 39 & 3.160 & 1,39 \\
\hline 40,2 & 3.180 & 1,81 \\
\hline 40 & 3.200 & 2,3 \\
\hline 39 & 3.250 & 1,59 \\
\hline 41 & 3.260 & 1,58 \\
\hline 40 & 3.270 & 0,68 \\
\hline 40 & 3.335 & 0,54 \\
\hline 40,2 & 3.360 & 2,05 \\
\hline 40 & 3.450 & 0,51 \\
\hline 40,1 & 3.600 & 2,25 \\
\hline 40,1 & 3.640 & 1,89 \\
\hline 39,6 & 3.660 & 3,03 \\
\hline 40 & 3.680 & 1,08 \\
\hline 40,6 & 3.770 & 1,71 \\
\hline
\end{tabular}

HOMA-IR: modelo homeostático para evaluar la resistencia a la insulina

Cuadro 5. Correlación entre el peso neonatal y el índice HOMA-IR

\begin{tabular}{lc}
\hline Peso neonatal & HOMA-IR $(\mathbf{m m o l} / \mathbf{L} \times \mathbf{\mu U I} / \mathbf{m l} / \mathbf{k})$ \\
\hline Coeficiente de correlación & 0,430 \\
$\mathrm{p}$ & 0,012 \\
$\mathrm{n}$ & 36 \\
\hline
\end{tabular}

el transporte y el metabolismo de glucosa en el músculo esquelético y en los adipocitos, en tanto que aumenta la gluconeogénesis en el hígado, por lo cual, en un primer momento, hay hipersecreción de insulina como mecanismo de compensación; posteriormente, esto altera la función de las células beta pancreáticas (como en el caso de mujeres gestantes con obesidad y diabetes mellitus de la gestación) (25).

En cuanto a las principales correlaciones entre los biomarcadores séricos, se encontró una correlación entre la leptina y la insulina $(r=0,6 ; p<0,000)$, $y$ entre la leptina y el índice HOMA-IR ( $r=0,5$; $p<0,000)$. En el estudio de Eriksson, et al., en Suecia, y el de Walsh, et al., en Irlanda, se obtuvieron estos mismos resultados $(6,18)$.

La leptina es producida por los adipocitos, cuyo aumento de tamaño lleva a la producción de factores proinflamatorios que inhiben la señalización del receptor de insulina y provocan la resistencia a esta, lo cual se compensa con la producción de mayores cantidades de esta hormona.

Una de las adolescentes gestantes, cuyos datos se descartaron del análisis final porque desarrolló eclampsia, presentó obesidad en el inicio del embarazo. La obesidad en el embarazo se ha asociado con la diabetes de la gestación, la eclampsia y el parto prematuro (26).

El exceso de nutrientes durante la gestación puede producir cambios epigenéticos que provocan modificaciones del fenotipo a largo plazo y predisponen a enfermedades crónicas como la obesidad, la resistencia a la insulina y la diabetes mellitus de tipo 2 (27). La ingestión inadecuada de nutrientes durante el embarazo se asocia con cambios en la metilación del promotor del gen de la leptina y en la producción de dicha hormona por la placenta $(28,29)$.

La leptina está involucrada en el adecuado desarrollo y maduración de diferentes órganos, como el páncreas (30). La metilación, un mecanismo epigenético, ocurre también en tejidos fetales. En modelos animales se ha demostrado que el exceso de ingestión de algunos macronutrientes durante la gestación, se asocia con cambios en la metilación de las regiones promotoras del gen de la leptina, su receptor y la adiponectina en la progenie (31).

La relación entre la metilación de promotores y las enfermedades metabólicas es importante, tal como se evidenció en un estudio con monocitos circulantes en adolescentes mexicanos, en el 
que se observó que los sujetos con obesidad y resistencia a la insulina presentaban disminución de la metilación de los promotores del gen de la leptina y del gen de adiponectina (32). Asimismo, en un trabajo con monocitos circulantes de personas europeas con obesidad, se evidenció una asociación inversa entre el índice HOMA-IR y la metilación del gen del supresor de señalización de las citocinas (SOCS), molécula que participa en las vías intracelulares de señalización de la insulina y de la leptina (33).

En el presente estudio, se encontraron correlaciones negativas significativas entre la adiponectina, el índice HOMA-IR y la insulina $(r=-0,4 ; p=0,017$ y $r=-0,4 ; p=0,025$, respectivamente), resultados similares a los descritos por Eriksson, Walsh y Lacroix en Canadá, en el primer trimestre de gestación de mujeres adultas $(6,18,34)$.

La adiponectina es una adipocitocina que aumenta la sensibilidad a la insulina y cuyos niveles disminuyen progresivamente durante la gestación; sus concentraciones están inversamente relacionadas con la ganancia de peso en el embarazo $(7,35)$. Es más, la disminución de los valores séricos de la adiponectina en la segunda mitad de la gestación, coincide con el estado de resistencia a la insulina característico del embarazo, como lo demuestran los resultados del estudio de Kac, et al. (17).

No se registró un peso superior a los $4.000 \mathrm{~g}$ en ningún recién nacido. Cinco de los neonatos presentaron pesos por debajo de los $2.500 \mathrm{~g}$, y dos de ellos fueron prematuros. En este estudio no se determinaron los niveles de leptina en los recién nacidos, pero en investigaciones de Japón, Estados Unidos y Egipto, se han encontrado concentraciones circulantes bajas de esta hormona en los neonatos con retardo del crecimiento intrauterino (36-38). Tales concentraciones podrían tener efectos epigenéticos en el desarrollo de los circuitos hipotalámicos de apetito y saciedad, y predisponer a la hiperfagia, a la disminución del gasto energético y a la obesidad en la edad adulta (30).

En madres adolescentes sanas sin obesidad ni antecedentes de dislipidemia, se observó una correlación positiva significativa entre el índice HOMA-IR y el peso neonatal $(r=0,43 ; p=0,012)$, lo cual podría ser el resultado de la resistencia a la insulina que se presenta a lo largo del embarazo. Si desde el inicio de la gestación el índice HOMA$\mathrm{IR}$ es elevado, sus valores aumentarán progresivamente $y$, cuanto mayor sea la resistencia a la insulina, mayor será el grado de transferencia fetoplacentaria de nutrientes, principalmente de glucosa.

En el estudio de Yamashita, et al., en Japón, se estudió el índice HOMA-IR en el segundo y el tercer trimestres de gestación de mujeres no diabéticas y se observó la misma correlación con el peso neonatal (39); sin embargo, en el presente estudio se registró este resultado desde el primer trimestre de gestación.

En el estudio de Crume, et al., en Estados Unidos, se obtuvieron resultados similares a los de la presente investigación. Dichos autores hallaron una correlación lineal entre el índice HOMA-IR de la primera mitad de la gestación en mujeres entre 21 y 33 años y el porcentaje de masa grasa neonatal (10), en tanto que en este, se observó que el comportamiento metabólico de las adolescentes gestantes fue similar al de las mujeres adultas embarazadas.

Los resultados de varios estudios han confirmado las relaciones entre el perfil lipídico materno y el peso del recién nacido (40). En el presente estudio no se encontraron correlaciones entre las concentraciones de colesterol total y de triglicéridos, y el peso neonatal. En trabajos previos en Irán y en India, se ha evidenciado una relación positiva del peso neonatal con los niveles séricos de triglicéridos de la madre, determinados en el tercer trimestre del embarazo $(41,42)$. En un estudio en Estados Unidos, se describió una correlación positiva entre los niveles de colesterol total medidos en los tres trimestres de gestación y el peso del recién nacido (43).

Por otra parte, en un estudio llevado a cabo en Ámsterdam, se observaron diferencias en los niveles de colesterol total y triglicéridos en el inicio del embarazo de mujeres de distintos grupos étnicos (44). Los resultados de las mediciones del perfil lipídico en el primer trimestre de gestación de adolescentes en dicho estudio, y los datos disímiles reportados en la literatura médica, corroboran que la determinación de los niveles de lípidos en la primera mitad del embarazo no es de utilidad para predecir el peso neonatal (43).

En este estudio se encontró una correlación significativa entre las concentraciones de adiponectina y las de colesterol total y colesterol HDL, y no se observaron relaciones entre la leptina y el perfil lipídico. En un trabajo previo en Alemania, en el que se midieron los niveles de adiponectina y el 
perfil lipídico en las semanas de gestación 16, 22, 30 y 36 , se encontró una correlación negativa entre los niveles de adiponectina y los de triglicéridos en los diferentes tiempos, así como una tendencia hacia la correlación positiva entre la adiponectina y el colesterol HDL de significación estadística solo en la semana 36 de embarazo, en tanto que no se evidenció una relación entre la adiponectina, el colesterol total y el LDL (45). En otra investigación en población mexicana en la que se midieron los indicadores bioquímicos en los tres trimestres del embarazo, en el segundo trimestre, se encontró una correlación negativa de la adiponectina con los triglicéridos, y una positiva de la leptina con el colesterol total y el HDL (46). Los hallazgos dispares de las investigaciones podrían deberse a diferencias en las características médicas y étnicas de las mujeres gestantes y los distintos momentos del embarazo en los que se tomaron las muestras de sangre.

Los resultados del presente estudio en adolescentes gestantes de 14 a 17 años residentes en Bogotá, aportan datos para una mejor comprensión del comportamiento metabólico durante el embarazo en la adolescencia. A partir de las mediciones realizadas, así como de la obtención de las medidas antropométricas neonatales, se establecieron correlaciones que permiten un mejor conocimiento de los efectos hormonales de la leptina, la adiponectina y la insulina, y del índice HOMA-IR en el primer trimestre de gestación en el recién nacido.

Este es un estudio piloto del cual se puede partir para próximos análisis que profundicen en la fisiología de la gestación en mujeres adolescentes, con un número mayor de participantes y exploración de los niveles de las adipocitocinas en cada uno de los trimestres de la gestación. Además, sería importante determinar si las adolescentes gestantes continúan o no su crecimiento, y hacer un seguimiento de la dieta para obtener conclusiones más amplias que permitan determinar el comportamiento hormonal, el aporte calórico y los efectos del crecimiento materno en el neonato.

\section{Agradecimientos}

Al Laboratorio de Lípidos y Diabetes, por el préstamo de los equipos requeridos para las mediciones antropométricas y bioquímicas. A las instituciones de salud que permitieron la captación de las adolescentes gestantes, así como a su equipo médico. A Andryu Mendoza, por su asesoría estadística para el análisis e interpretación de los resultados.

\section{Conflicto de intereses}

Las autoras declaran no tener conflictos de intereses relacionados con el desarrollo de esta investigación.

\section{Financiación}

El Departamento Administrativo de Ciencia, Tecnología e Innovación, Colciencias, brindó apoyo económico a la investigadora principal mediante la Convocatoria Nacional de Jóvenes Investigadores e Innovadores, 2015. El Departamento de Ciencias Fisiológicas de la Facultad de Medicina de la Universidad Nacional de Colombia, financió los insumos para la obtención y el procesamiento de las muestras.

\section{Referencias}

1. Colombiana de Salud, S.A. Guía de atención: Morbilidad materna. Manejo de la obesidad materna antes, durante y después de la gestación. Junio de 2013. 2013. Fecha de consulta: 25 de marzo de 2017. Disponible en: http://www. colombianadesalud.org.co/GUIAS_MATERNO_INFANTIL/ OBESIDAD\%20Y\%20EMBARAZO.pdf

2. Escudero VL, Parra SB, Herrera MJ, Restrepo MS, Zapata LN. Estado nutricional del hierro en gestantes adolescentes: Medellín, Colombia. Rev Fac Nac Salud Pública. 2014;32:71-9.

3. Ministerio de Salud y Protección Social, Profamilia. Encuesta Nacional de Demografía y Salud. Componente demográfico. ENDS Colombia, 2015. Bogotá; 2015. p. 1-430. Fecha de consulta: 11 de marzo de 2017. Disponible en: http://profamilia.org.co/docs/ENDS\%20\%20TOMO\%20I.pdf

4. Fondo de Población de Naciones Unidas -UNFPAAmérica Latina y el Caribe. América Latina y el Caribe tienen la segunda tasa más alta de embarazo adolescente en el mundo. Fecha de consulta: 11 de marzo de 2017. Disponible en: http://lac.unfpa.org/es/news/ am\%C3\%A9rica-latina-y-el-caribe-tienen-la-segunda-tasam\%C3\%A1s-alta-de-embarazo-adolescente-en-el-mund-1

5. Guevara-Núñez SP. Aspectos nutricionales de la gestación. En: Parra Pineda MO, Angel Müller E, editores. Obstetricia integral siglo XXI. Primera edición. Bogotá: Universidad Nacional de Colombia; 2009. p.136-61.

6. Eriksson B, Löf M, Olausson H, Forsum E. Body fat, insulin resistance, energy expenditure and serum concentrations of leptin, adiponectin and resistin before, during and after pregnancy in healthy Swedish women. Br J Nutr. 2010; 103:50-7. https//doi.org/10.1017/S0007114509991371

7. Miehle K, Stepan H, Fasshauer M. Leptin, adiponectin and other adipokines in gestational diabetes mellitus and preeclampsia. Clin Endocrinol (Oxf). 2012;76:2-11. https//doi. org/10.1111/j.1365-2265.2011.04234.x

8. Scholl TO, Stein TP, Smith WK. Leptin and maternal growth during adolescent pregnancy. Am J Clin Nutr. 2000; 72:1542-7. https://doi.org/10.1093/ajcn/72.6.1542

9. Valdés ER, Lattes KA, Muñoz HS, Barja PY, Papapietro KV. First-trimester adiponectin and subsequent development of preeclampsia or fetal growth restriction. Gynecol Obstet Invest. 2011;72:152-6. https//doi.org/10.1159/000328418 
10. Crume TL, Shapiro AL, Brinton JT, Glueck DH, Martínez M, Kohn M, et al. Maternal fuels and metabolic measures during pregnancy and neonatal body composition: The Healthy Start Study. J Clin Endocrinol Metab. 2015;100: 1672-80. https//doi.org/10.1210/jc.2014-2949

11. Costa e Silva LI, Gomes FM, Valente MH, Escobar AM, Brentani AV, Grisi SJ. The intergenerational effects on birth weight and its relations to maternal conditions, Sao Paulo, Brazil. Biomed Res Int. 2015;2015:615034. https:// doi.org/10.1155/2015/615034

12. República de Colombia, Ministerio de Salud. Resolución número 00002465 de 2016. Fecha de consulta: 4 de marzo de 2017. Disponible en: https://www.minsalud.gov. co/Normatividad_Nuevo/Resolucion\%202465\%20de\%20 2016.pdf

13. Ministerio de Salud y Protección Social, Colciencias. Guía de práctica clínica del recién nacido prematuro. Sistema General de Seguridad Social en Salud - Colombia. 2013 Guía 04. p.1-512. Fecha de consulta: 16 de abril de 2017. Disponible en: https://www.minsalud.gov.co/sites/rid/ Lists/BibliotecaDigital/RIDE/INEC/IETS/GPC_Completa_ Premat.pdfojrteeriuieruieriure

14. Battaglia FC, Lubchenco LO. A practical classification of newborn infants by weight and gestational age. J Pediatr. 1967;71:159-63.

15. Chandrasekaran S, Neal-Perry G. Long-term consequences of obesity on female fertility and the health of the offspring. Curr Opin Obstet Gynecol. 2017;29:180-7. https// doi.org/10.1097/GCO.0000000000000364

16. Chandi A, Sirohiwal D, Malik R. Association of early maternal hypertriglyceridemia with pregnancy-induced hypertension. Arch Gynecol Obstet. 2015;292:1135-43. http//doi.org/10.1007/s00404-015-3706-1

17. Kac G, dos Santos Vaz J, Schlüssel MM, Moura AS.Creactive protein and hormones but not IL-6 are associated to body mass index in first trimester of pregnancy. Arch Gynecol Obstet. 2011;284:567-73. https//org/10.1007/ s00404-010-1573-3

18. Walsh JM, Byrne J, Mahony RM, Foley ME, McAuliffe FM. Leptin, fetal growth and insulin resistance in nondiabetic pregnancies. Early Hum Dev. 2014;90:271-4. Early Hum Dev. 2014;90:271-4. https//doi.org/10.1016/j. earlhumdev.2014.03.007

19. Münzberg $\mathbf{H}$, Morrison $\mathbf{C D}$. Structure, production and signaling of leptin. Metabolism. 2015;64:13-23. https/doi. org//10.1016/j.metabol.2014.09.010

20. Tessier DR, Ferraro ZM, Gruslin A. Role of leptin in pregnancy: Consequences of maternal obesity. Placenta. 2013; 34:205-11. https//doi.org/10.1016/j.placenta.2012.11.035

21. Zhao Y, Li Q, Li YC. Effects of body mass index and body fat percentage on gestational complications and outcomes. J Obstet Gynaecol Res. 2014;40:705-10. Fecha de consulta: 25 de marzo de 2018. Disponible en: http://www. placentajournal.org/article/S0143-4004(12)00470-5/pdf

22. Cinti S, Mitchell G, Barbatelli G, Murano I, Ceresi E, Faloia $\mathbf{E}$, et al. Adipocyte death defines macrophage localization and function in adipose tissue of obese mice and humans. J Lipid Res. 2005;46:2347-55. Fecha de consulta: 20 de marzo de 2018. Disponible en: http://www. jlr.org/content/46/11/2347.long
23. Hotamisligil GS, Budavari A, Murray D, Spiegelman BM. Reduced tyrosine kinase activity of the insulin receptor in obesity-diabetes. Central role of tumor necrosis factoralpha. J Clin Invest. 1994;94:1543-9. https//doi.org/10.1172/ JCl117495

24. Schenk S, Saberi M, Olefsky JM. Insulin sensitivity: Modulation by nutrients and inflammation. Clin Invest. 2008; 118:2992-3002. https//doi.org/10.1172/JCI34260

25. Mlinar B, Marc J. New insights into adipose tissue dysfunction in insulin resistance. Clin Chem Lab Med. 2011;49: 1925-35. https//doi.org/10.1515/CCLM.2011.697

26. Marchi J, Berg M, Dencker A, Olander EK, Begley C. Obesity in pregnancy - a review of reviews. Obes Rev. 2015;16:621-38. https://doi.org/10.1111/obr.12288

27. Hoffman DJ, Reynolds RM, Hardy DB. Developmental origins of health and disease: current knowledge and potential mechanisms. Nutr Rev. 2017;75:951-70. https:// doi.org/10.1093/nutrit/nux053

28. Misra VK, Trudeau S. The influence of overweight and obesity on longitudinal trends in maternal serum leptin levels during pregnancy. Obesity. 2011;19:416-21. https// doi.org/10.1038/oby.2010.172

29. Schanton M, Maymó JL, Pérez-Pérez A, SánchezMargalet V, Varone CL. Involvement of leptin in the molecular physiology of the placenta. Reproduction. 2018; 155:R1-12. https//doi.org/10.1530/REP-17-0512

30. Briffa J, McAinch A, Romano T, Wlodek M, Hryciw DH. Leptin in pregnancy and development: A contributor to adulthood disease? Am J Physiol Endocrinol Metab. 2015; 308:E335-50. https://doi.org/10.1152/ajpendo.00312.2014

31. Khalyfa A, Carreras A, Hakim F, Cunningham JM, Wang Y, Gozal D. Effects of late gestational high-fat diet on body weight, metabolic regulation and adipokine expression in offspring. Int J Obes. 2013;37:1481-9. https://doi.org/10. 1038/ijo.2013.12

32. García-Cardona MC, Huang F, García-Vivas JM, LópezCamarillo C, Del Río-Navarro BE, Navarro-Olivos E, et al. DNA methylation of leptin and adiponectin promoters in children is reduced by the combined presence of obesity and insulin resistance. Int J Obes. 2014;38:1457-65. https:// doi.org/10.1038/ijo.2014.30

33. Ali O, Cerjak D, Kent JW, James R, Blangero J, Carless MA, et al. Methylation of SOCS3 is inversely associated with metabolic syndrome in an epigenome-wide association study of obesity. Epigenetics. 2016;11:699-707. https://doi. org/10.1080/15592294.2016.1216284

34. Lacroix M, Battista M-C, Doyon M, Ménard J, Ardilouze J-L, Perron P, et al. Lower adiponectin levels at first trimester of pregnancy are associated with increased insulin resistance and higher risk of developing gestational diabetes mellitus. Diabetes Care. 2013;36:1577-83. https:// doi.org/10.2337/dc12-1731

35. Aye ILMH, Powell TL, Jansson T. Review: Adiponectin-The missing link between maternal adiposity, placental transport and fetal growth? Placenta. 2013;34(Suppl.):S40-5. https:// doi.org//10.1016/j.placenta.2012.11.024

36. Mise H, Yura S, Itoh H, Nuamah M, Takemura M, Sagawa $\mathbf{N}$, et al. The relationship between maternal plasma leptin levels and fetal growth restriction. Endocr J. 2007;54:94551. https://doi.org/10.1507/endocri.K06-225 
37. Catov JM, Patrick TE, Powers RW, Ness RB, Harger G, Roberts JM. Maternal leptin across pregnancy in women with small-for-gestational-age infants. Am J Obstet Gynecol. 2007;196:558. https://doi.org/10.1016/j.ajog.2007.01.032

38. Nezar MA, El-Baky AM, Soliman OA, Abdel-Hady HA, Hammad AM, Al-Haggar MS. Endothelin-1 and leptin as markers of intrauterine growth restriction. Indian J Pediatr. 2009;76:485-8. https://doi.org/10.1007/s12098-009-0079-0

39. Yamashita H, Yasuhi I, Fukuda M, Kugishima Y, Yamauchi Y, Kuzume A, et al. The association between maternal insulin resistance in mid-pregnancy and neonatal birthweight in uncomplicated pregnancies. Endocr J. 2014; 61:1019-24. https://doi.org/10.1507/endocrj.EJ14-0163

40. Barrett HL, Nitert MD, McIntyre HD, Callaway LK. Normalizing metabolism in diabetic pregnancy: Is it time to target lipids? Diabetes Care. 2014;37:1484-93. https://doi.org/10. 2337/dc13-1934

41. Mossayebi E, Arab Z, Rahmaniyan M, Almassinokiani F, Kabir A. Prediction of neonates' macrosomia with maternal lipid profile of healthy mothers. Pediatr Neonatol. 2014;55:28-34.

42. Kulkarni SR, Kumaran K, Rao SR, Chougule SD, Deokar TM, Bhalerao A, et al. Maternal lipids are as important as glucose for fetal growth: Findings from the Pune Maternal Nutrition Study. Diabetes Care. 2014;36:2706-13. https:// doi.org/10.2337/dc12-2445
43. Boghossian NS, Mendola P, Liu A, Robledo C, Yeung EH. Maternal serum markers of lipid metabolism in relation to neonatal anthropometry. Journal Perinatol. 2017;37:62935. https://doi.org/10.1038/jp.2017.22

44. Schreuder YJ, Hutten BA, van Eijsden M, Jansen EH, Vissers MN, Twickler MT, et al. Ethnic differences in maternal total cholesterol and triglyceride levels during pregnancy: The contribution of demographics, behavioural factors and clinical characteristics. Eur J Clin Nutr. 2011; 65:580-9. https://doi.org/10.1038/ejcn.2010.282

45. Ritterath C, Rad NT, Siegmund T, Heinze T, Siebert G, Buhling KJ. Adiponectin during pregnancy: Correlation with fat metabolism, but not with carbohydrate metabolism. Arch Gynecol Obstet. 2010;281:91-6. https://doi.org/10.1007/ s00404-009-1087-z

46. Perichart-Perera O, Muñoz-Manrique C, Reyes-López A, Tolentino-Dolores M, Espino y Sosa S, RamírezGonzález MC. Metabolic markers during pregnancy and their association with maternal and newborn weight status. PLoS One. 2017;12:1-12. https://doi.org/10.1371/journal. pone. 0180874 